\title{
Plus d'un
}

\author{
Jean-Luc NANCY
}

Universidad de Estrasburgo escrituraeimagen@filos.ucm.es

\section{Resumen}

«Más de uno» fue una de las expresiones más queridas de Derrida. A partir de esta fórmula, propuesta como título para este trabajo, Jean-Luc Nancy reflexiona sobre la naturaleza de ese más de uno para mostrar cómo uno en el uno más uno de la serie de los números, no puede ser entendido como el origen de la monótona sucesión lineal de la operación aritmética: 1, 2, 3, 4... La adición constituye también la diferencia consigo en la que el uno es desplazado fuera de sí y, de ese modo, «uno» tiene lugar como ritmo. Una rítmica pone en juego el propio estatuto del uno y de la numeración. El uno que se excede, el uno excedente se trastorna en la unidad de una pulsación, en la simplicidad absoluta de un envío, de un transporte. Afrontamos, por tanto, una lógica distinta de la unidad en la que «uno» no se deja conocer ni contar.

Palabras clave: Repetición, ritmo, uno y más de uno, adición y multiplicación, división y separación, unidad innumerable.

\begin{abstract}
"More than one" was one of Derrida's dearest expressions. Based on this formula, which is the title of this paper, Jean-Luc Nancy reflects on the nature of that more than one to show how one in the one plus one of the number series cannot be taken to be the origin of the monotonous linear succession of the arithmetic operation: $1,2,3,4, \ldots$ The addition also constitutes the difference with itself in which one is displaced out of itself, and in this way, "one" exists as rhythm. A rhythm puts
\end{abstract}


into play the very statute of one and the numbering. The one that exceeds itself, the exceeding one upsets itself into the unit of a pulsation, into the absolute simplicity of one envoy, of one transport. We face, therefore, a different logic from that of the unit, in which "one" does not allow itself to be known nor counted.

Key words: Iterability, repeatibility, rhythm, one and more than one, addition and multiplication, division and separation, innumerable unit.

«[...] alors nous pourrons tantôt descendre en démembrant avec une force titanesque l'un dans le multiple, tel Osiris, tantôt monter en rassemblant avec une force apollinienne le multiple dans l'un, comme s'il s'agissait des membres d'Osiris. "

Pic de la Mirandole1

« [...] le double ne s'ajoutait pas seulement au simple. Il le divisait et le suppléait. Il y avait aussitôt une double origine plus sa répétition. Trois est le premier chiffre de la répétition. Le dernier aussi, car l'abîme de la représentation reste toujours dominé par son rythme, à l'infini. L'infini n'est sans doute ni un, ni nul, ni innombrable. Il est d'essence ternaire. " 2

Un, deux et la reprise de cette division et de cette addition. Reprise qui compte pour un de plus, et qui fait trois. Le deux divise l'un et le supplée : 1'un n'a pas eu lieu, il n'a eu lieu qu'en se dédoublant et se répétant.

L' « abîme de la représentation », c'est la non-présence qui se rejoue à chaque nouvelle proposition de signification : un signe, dès qu'il fait signe, renvoie à un autre signe et leur renvoi ne renvoie ou ne réfère à rien (à rien comme une « chose », une « présence », un « donné »). Signe et signe et rien, tel est le rythme.

Mais rien - nulle chose aucune chose, pas de « un »- nous commençons à savoir que c'est « quelque chose », comme le veut la langue française (res, rem, un rien). Pour comprendre quelle « chose » est rien, si cela peut se comprendre, peutêtre faut-il considérer comment s'y fait le renvoi ou la référence : par le rythme. Autrement dit, le nihilisme consiste à tenir que nul signe ne renvoie à une chose et que les signes n'enchaînent que cette nullité. Il faut pour cela tenir que « un » correspond à « un », à un « un ». Mais si la répétition des signes - le langage, et plus que lui, la signifiance de toutes choses - vaut comme rythme, la scène n'en est-elle

1 De la Dignité de l'homme, trad. Yves Hersant, Editions de l'Eclat, 1983, http://www.lybereclat.net/lyber/mirandola/pictrad.html

2 Jacques Derrida, L'Écriture et la différence, Paris, Seuil, 1967, p. 435. 
pas changée ? Le rythme met en jeu plus d'un. Plus d'un, plus qu'un, « ni un, ni nul, ni innombrable » et rien, la chose, en forme d'infini. Sortie du nihilisme. Voilà de quoi nous nous occupons ici.

$$
* * *
$$

Le dé- du dédoublement ou le ré- de la répétition font « un » de plus - un qui n'est pas un autre un ajouté dans la série indéfinie des unités, mais un dont l'addition est aussi l'écart de un à deux, cet écart qui divise un en même temps qu'il le supplée, c'est-à-dire qu'il tient sa place et son rôle et que, ce faisant, il ajointe l'un à lui-même - le « un » simple au « un » dédoublé. Cet ajointement par la division, cette flexion, cette articulation transforme l'ajout monotone de un à un en rythme. C'est-à-dire qu'à la succession linéaire se substitue le retour du même - ce retour avec lequel Nietzsche a ouvert ce qu'on pourrait nommer l'époque de «plus d'un ». Le «même » en effet n'est pas l' "un »-du moins comme identique. Il est ce qui pour être même ou semblable (das Gleiche) ne doit justement pas être « un ». Ou doit entraîner l'un - avec sa propre unité et unicité - dans un déplacement dont nous sommes en train de commencer à éprouver le mouvement, tout en éprouvant qu'en réalité c'est depuis le commencement de notre histoire que l' "un » se déplace, en lui-même ou hors de lui-même. Nous allons avoir des raisons et des occasions d'y revenir, sans pour autant prétendre reconstituer - ce qui serait la tâche d'un immense traité - l'histoire de l'un, cette histoire qui en somme est une depuis Héraclite jusqu'à Derrida et à nous - mais qui est une sur le mode d'avoir d'entrée de jeu déplacé, déporté, débordé aussi bien sa propre unité que 1' « un » qu'elle semblait avoir en charge.

(Pour ne pas en dire plus maintenant, qu'il suffise un instant de se remémorer quelques éclairs de l'un d'Héraclite, de celui de Platon, de ceux d'Aristote ou de Plotin, de l'unité de la Trinité augustinienne puis de celle du Dieu d'Averroès, de celui de Thomas, sans parler d'Ibn Arabi ou d'Eckhart, de la monade de Leibniz, de l'Un-pour-soi de Hegel ou de l'unique de Stirner. Ces quelques mentions sont peu de choses, on le sait, dans le cours immense et proliférant de cette histoire que peutêtre un jour on pourra caractériser comme l'histoire de l'Un, l'unique histoire de $l^{\prime}$ Un, de son avènement et de ses avatars ${ }^{3}$ - « un jour » qui sera un jour tel que l'Un

\footnotetext{
${ }^{3}$ Le seul de nos contemporains qui ait entendu édifier en propres termes une « ontologie »- Alain Badiou - la nomme « ontologie du multiple » et la place sous le signe de la proposition que « l'Un n'est pas ». Il prend encore ainsi le relais de cette histoire qu'en même temps il interrompt en opposant au ne-pas-être de l'Un le non-être de l'événement. Chez Derrida, le (dé)doublement de l'origine ouvre de l'événement dans l'être ou bien plutôt à la place laissée libre par la Destruktion heideggérienne de toute ontologie. L'un n'a pas à être ou à n'être pas si l'être lui-même n'est pas. Mais il arrive, comme rythme. C'est-à-dire aussi qu'il s'arrive à lui-même, il se survient - comme « plus d'un ».
} 
s'y sera désuni de lui-même. En fait nous y sommes-nous déjà. C'est précisément de quoi nous parlons.)

«Plus d'un », ce fut en des temps postérieurs au texte que j'ai cité, une expression favorite de Derrida. Je pourrais dire : une expression-fétiche. Plus d'une langue, plus d'une séance, plus d'une loi, etc. - et peut-être plus d'un « un » : qu'il ait ou non risqué ce tour, il est prescrit en droit. Prescrit par la logique du supplément d'origine, pour reprendre ses termes, mais prescrit à cette logique elle-même depuis le plus ancien départ (y en a-t-il eu un, un seul ?) de la philosophie.

Plus d'une philosophie ? Plus d'une, la philosophie, elle-même ? L'unité de la philosophie n'a pas cessé d'être pour elle-même objet de litige. Tantôt il faut qu'il $\mathrm{y}$ ait de manière essentielle plusieurs philosophies qui divergent ou qui s'affrontent, tantôt il faut qu'il y ait une philosophie qui se poursuit et se reprend à moins qu'elle n'en vienne à prononcer sa propre « fin » et son ouverture à une autre «pensée ». Mais la philosophie elle-même présuppose la mise à distance d'un principe unique de la sophia dont elle parle. Le philein est principe, lui, de non-unité : il implique la possibilité de variations, de distances et d'approches, et par principe donc il tient à distance l'unité et l'unicité qu'on tendrait toujours obstinément à supposer à une sophia.

Cela veut dire aussi que toujours et sans cesse, d'une manière ou d'une autre, la philosophie est plus que la philosophie. Plus d'une et plus qu'une. Plus qu'ellemême : c'est aussi cela que veut indiquer philein. Par où nécessairement et originairement la philosophie mettrait en jeu un excès sur l'unité : la sienne comme celle de son thème (qu'il se nomme « être » ou comme on voudra; ici précisément ne compte pour finir que l'excès de toute signification ou signifiance sur un sens quel qu'il soit). « Sens unique »-c'est aussi un des noms majeurs de notre histoire en tant que processus et que mise en cause du processus.)

Il est permis d'apercevoir une asymptote commune aux deux façons de procéder : celle selon laquelle l'un serait son propre excès. De manière analogue, cette fois, d'une part à l'Ereignis de Heidegger (qui est " appropriation » du non-un du propre sur le fond de l' " unicité » de l'être - Einzigkeit des Seyns qui apparaît p.ex. dans Beiträge, 12 ) d'autre part à l' " univocité de l'être " de Deleuze (qui n'est faite que de différent/ciations). - L'espèce de mise en réseau à laquelle je procède ainsi de manière hâtive ne vise pas à rassembler toutes ces pensées - sinon, malgré tout, dans la perspective d'une question comme celle-ci : qu'est-ce donc qu'une époque ? Où se joue l'unité d'un moment, d'un stade ou d'un palier? Il n'y a jamais pure disparité hétérogène entre les pensées d'une époque même si on sait mal en quoi elle fait « époque » (donc " une ») et même s'il y a en même temps des discordances sensibles. "Une époque », ce n'est pas un consensus, mais c'est jusque dans le dissensus le plus vif une relative possibilité de renvoyer les uns aux autres. 
« Plus d'un » : cela veut dire à l'évidence plus d'une chose (et qu'est-ce que « une chose » en ce sens particulièrement indéterminé ? un signifié, un référent, un objet, un concept? en vérité cela veut dire simplement « un », une unité quelconque, le fait qu'on puisse remplir l'intention de " vouloir dire » par autre chose que par « rien »- ceci donc simplement que « un » ce n'est pas rien, et que pour cette raison la question minimale de l' « un » est la question du nihilisme : rien, ou de l'un).

«Plus d'un » veut dire avant tout «plus que un seul», plus nombreux, cela veut dire en ce sens le nombre même, ou la numération. Plus d'un : un, deux, trois, quatre... (succession non rythmique). Immédiatement cela entraîne pluralité des « un » : le un-un est suivi d'un deuxième un, puis d'un autre. Mais la pluralité des « un » ouvre aussitôt sur la question de sa nature : est-elle addition, multiplication ou bien distinction, dissimilitude?

Si la pluralité est d'addition ou de multiplication, «plus d'un » peut s'étendre indéfiniment comme la série des nombres, de toutes les numérations possibles (nombres « naturels », « réels », «imaginaires », « irrationnels », etc. ). Le principe est celui de la numération ou de la numérosité : plus d'un, c'est-à-dire non pas seulement quelques uns mais beaucoup. Ou plus précisément, il n’y a jamais « quelques uns » sans que « beaucoup » soit à l'horizon. Beaucoup, la multitude, c'est-à-dire la multiplication des uns non reconduits à la juridiction d'un Un, tout simplement parce qu'il n'y a pas de juridiction ni de « Un » majuscule mais seulement l'énumération des « uns ». C'est l'énumération qui est le principe, la foule, le nombreux dont le nombre ne cesse de croître. L'addition reconduit toujours plus loin la somme indéfinie qui ne fera jamais elle-même une unité.

Lorsque l'humanité en vient à compter sept milliards d'individus - neuf milliards dans quarante ans, affirment les projections - le grand nombre semble disséminer en lui toute unité supposée de l' " homme », du « genre humain » qui, de fait, s'avère beaucoup plus comme le genre d'une multiplication indéfinie que comme celui de la croissance d'une quelconque unité générique. Et qui pourrait donc s'avérer comme le genre d'aucun genre, comme un brouillage des genres ou plus exactement des espèces puisque homo sapiens est une espèce du genre homo. Espèce qui ne se laisse pas saisir comme telle, c'est-à-dire spécifier ou saisir sous un aspect qui lui serait propre : l'aspect qui est en somme un terme jumeau de « espèce ». L'espèce sans aspect, ou l'espèce dont la caractéristique se réduirait à ce qui définit en principe l'« espèce » zoologique, à savoir la fécondité interne. L'espèce humaine ne développerait pas d'autre aspect que sa propre reproduction exponentielle accompagnée d'une réduction ou d'une destruction de beaucoup d'autres espèces vivantes et d'une transformation génétique de bien d'autres, voire d'elle-même à travers des procédés comme le clonage. Plus d'un, oui, jusqu'à neuf milliards, et pas un homme ? Peut-on imaginer cela : qu'une identité spécifique se déploie comme pure multiplication d'unités qui tendanciellement ne valent que 
comme unités de compte dans le dénombrement lui-même interminable ? Le sème ou la semence de «l'homme » disséminé dans sa pure dispersion?

La multiplication fait apparaître en même temps l'autre valeur du "plus d'un ». La dissémination - cet autre mot de Derrida - dérobe le sens (et le sème, et la semence) à l'unité et à l'unicité. « Plus d'un » c'est alors la multitude moins comme prolifération que comme efflorescence, comme surabondance et pour finir comme excès de sens.

Sans doute la croissance de la population humaine procède elle-même de l'activité de l'homme qui ne cesse de poursuivre autre chose que la reproduction de ses conditions de vie et de son espèce, frayant sans cesse la voie à des vies imprévues, non inscrites au tableau des espèces et dépassant de loin tout ce que les mutations peuvent introduire de nouveauté parmi les vivants. En tant que plus d'un et que plus d'une espèce, l'homme est lui-même une mutation du vivant : il transforme la vie, qui est entretien, en entreprise - s'il est permis de retenir le sens premier du mot, qui ne dit rien d'autre que «mise en œuvre ».

Tant que la vie s'entretient, elle répond à des rythmes, à des alternances de sommeil et de veille, d'action et de passion, de paroles échangées, de langues et de peuples. Lorsqu'elle transforme l'entretien en entreprise elle défait les rythmes, elle en crée certes de nouveaux, elle invente des cadences complexes, mais elle entraîne aussi toute cadence dans une accélération indéfinie. C'est un autre aspect du passage aux grands nombres : la population, la vitesse, les dimensions des bulles financières ou les mesures de l'univers, tout reçoit le signe du très grand nombre. C'est le très grand nombre que reçoit et que produit en même temps ce mouvement qu'on nomme «mondialisation » ou « globalisation » et qui sous l'un ou l'autre nom élève la présomption d'une unité. Mais l'unité " globale » ne peut pas être autre chose qu'une unité elle-même numérique, nombreuse, cumulative et dissociative en même temps, le "plus d'un » déplié en " un plus un plus un... ». Le " plus », par conséquent, comme signe d'addition et non comme indice de dépassement.

Dans l'addition, l'unité du tout n'est pas distincte de l'uniformité de l'opération additive. Or la valeur du « plus » change complètement entre « un plus un » et « plus d'un ». Le premier emploi se réduit à une écriture arithmétique $(+)$ et notre usage de la langue lui réserve d'ailleurs une prononciation distincte puisque le $s$ doit être prononcé tandis qu'il ne l'est pas dans les autres valeurs du mot.

Dans le second emploi, " plus d'un », il s'agit de passer au-delà de l'unité du un. Il ne s'agit pas seulement de pouvoir compter plusieurs « uns » ou plusieurs fois le même " un ». Ce qui est en jeu n'est pas plusieurs, ni beaucoup, mais le statut de l' „un » lui-même. Cette mise en jeu ne s'oppose pas pour autant tout uniment à l'addition et à la multiplication : le texte de Derrida dit avec précision « le double ne s'ajoutait pas seulement au simple ». L'addition n'est pas niée, elle est traversée par un autre mouvement : en passant de un à deux, et donc de un "un » à deux 
« un » je ne juxtapose pas seulement des unités - qui de surcroît sont nécessairement supposées identiques pour autant qu'on peut les ajouter l'une à l'autre - mais j'affecte aussi la nature ou l'état de l' " un » (et par conséquent non seulement du premier, mais du deuxième et de tout autre « un » à venir).

Cette affection est une division et une supplémentation ou une suppléance : il y a bien un donné un, suivi d'un deuxième un, mais de ce fait le premier " un " ne peut pas rester immobile en soi. D'une part il est lui-même passé dans le deuxième - à titre de " un », justement - et en cela il est divisé de lui-même. D'autre part, puisque cette division retire à l' " un » sa propriété d'unicité initiale, et puisque de cette manière on n'a pas vraiment commencé - on n'a pas vraiment compté « un plus un »-alors le commencement ou l'origine avère sa propriété à la place de cela qui aurait dû ou pu commencer, cela que nous sommes portés à représenter comme devant former le « un » du premier pas, alors même qu'un « pas », de lui-même, se divise.

On pourrait dire qu'il y a deux logiques de l' « un » : une qui le pose comme immobile, mais qui soulève la difficulté de savoir comment passer à plus d'un, et une qui le reçoit comme le pas lui-même, qui de soi se divise et supplée son initialité.

« Un » divisé n'est pas « un + un » mais deux « un » issus de la division d'un premier qui n'a pas eu lieu. Deux temps, donc, et la division elle-même comme initialité de ce qui n'a pas « commencé » et donc troisième temps. Ternaire dont la figure métrique pourrait être le mètre dit « amphibraque » - _ _ _ ce qui n'exclut pas les autres battues possibles, ni la possibilité de passer à quatre par dédoublement du second temps. Comme on le sait, c'est ce qui se produit dans la dialectique hégélienne, tout comme d'ailleurs la Trinité chrétienne qui peut être considérée comme l'unité de trois avec cette unité elle-même que nomme « Dieu » (ou bien, dans l'analogie humaine dont se sert Augustin, l'unité de mémoire, intelligence et amour avec la personne qui n'est elle-même que dans ces trois propriétés ensemble) - sans que pour autant le quatrième temps indique une quelconque « unité » de plus mais bien plutôt l'extension ou la tension interne du ternaire. Ainsi le Geviert de Heidegger est-il la figure de l'extension ou de l'ouverture du monde, extension dans laquelle l'existence est possible selon l'Ereignis qui peut être compris comme l'événement de l'un (de chaque un et/ou du un de l' "être ») et donc aussi bien comme sa division ou partition. ${ }^{4}$

\footnotetext{
4 Pour enchaîner du ternaire au quaternaire chez Derrida, il faudrait considérer comment il reçoit le Geviert dont il ne manque pas de rappeler qu'il se forme pour Heidegger «à partir d'une Unité originelle» (p. ex. La Dissémination, Paris, Seuil, 1972, p. 394).
} 
"Il faut que l'"un" se sépare de lui-même, se repousse, se condamne lui-même, qu'il s'abolisse au profit des autres pour se reconstituer dans leur unité avec lui.»5

Je n'entreprends pas, bien évidemment, de parcourir les figures et les concepts du trois et du quatre dans la philosophie, la théologie, la mystique, l'alchimie : je veux seulement rappeler combien cette numération a été importante à travers toute notre histoire. Depuis toujours, et bien avant la philosophie, c'est toute une numérologie ou une arithmosophie qui a recouvert ou doublé l'énumération. Le « un, un... » a toujours déjà donné lieu à une spéculation sur le " un » lui-même, le « un » qui ne s'énumère pas. Au-delà de toute numérologie, cependant, il s'agit d'entend$r e$ : car il y a une battue, un battement, il y a une pulsation.

Peut-être « un » n'est-il jamais possible sans cette pulsation, qui n'est pas numération et qui pourtant déjà joue dans la numération, qui déjà percute le simple « un + un + un... ». Il n'y a pas d'addition sans répétition ni de succession sans scansion. Pas de psalmodie de la récitation des tables - " un plus un, deux, deux plus un, trois... »-sans esquisse de chant, d'incantation. Ce n'est plus alors le compte qui vaut, c'est la résonance : c'est le langage du calcul se renvoyant à lui-même cela qui excède et calcul et langage.

«Un », peut-être, serait affaire de rythme et non de compte, et non de nombre du rythme $-3,4$, etc. - mais de mouvement, d'allure, d'allant et de pulsation. On a très longtemps disputé - en particulier entre les traditions platonicienne, aristotélicienne et néoplatonicienne - de la primauté entre l'un et l'être. C'est à ce débat que voulut mettre fin Pic de la Mirandole en écrivant son De Ente et Uno. Sa solution consiste à dépasser la rivalité des deux prétendants en les attribuant l'un et l'autre (avec le bien et le vrai) à Dieu, lequel ne se laisse pas saisir par la pensée 6 car son unité est « antérieure » à celle de l'un lui-même, elle est infinité en acte et en même temps intimité à elle-même telle que sa « connaissance vraie » est une « totale ignorance $»^{7}$.

En d'autres termes, il y a un et un. Il y a plus d'un enfoui au cœur de l'un - et de l'être. S'il est impossible, en effet, qu'un être soit sans être un, il est possible en revanche, il est même peut-être nécessaire que l'un comme l'être relèvent d'une autre logique de l'Un. En s'écartant de « l'être » substantif - qui en effet se soumet

\footnotetext{
5 André Breton, Les vases communicants, 1932.

6 Pic cite Anselme : «Domine, non solum es quo majus cogitari nequit; sed es quiddam majus quam cogitari possit.» (Proslogion $X V$ )

7 De Ente et Uno, cité à partir de l'édition du texte donnée par A. Festugière en 1932, dans Archives d'Histoire Doctrinale et Littéraire du Moyen Age vol. VII.
} 
d'emblée à la numération - pour approcher « être », le verbe, ce verbe qui n' " est pas » comme le dit Heidegger et qui pour cette raison appelle sa mise sous rature non pas une annulation, mais un écartement (un écartèlement si on veut, ou un étoilement) - on vient à penser ce verbe comme verbe transitif (Heidegger le demande parfois). Cette transitivité agrammaticale pourrait se rendre ainsi : être écarte l'être.

Etre alors n'est pas « un » être, ni la production d'étants un par un. Mais «être » forme l'acte d'écartement, de distinction et de division des étants. Cet acte est un sans être « un être ». Il est son unité d'acte, son unité transitive qui ne précède pas les " uns » des étants et qui ne leur survient pas non plus mais qui est ou plutôt qui agit, qui acte leur événement ou avènement.

Cet « un » ne se laisse pas compter. Il est l'un de la poussée, de la pulsation ou de la pulsion qui fait être ce qui est. Un rythmique, c'est-à-dire à la fois un composé, pluriel en soi, et un soustrait au dénombrement. On ne parle pas ici de compter les temps du rythme : on parle de le frapper dans ses mains ou sur une caisse, ce qui est tout autre chose. On parle de danse et de cadence. L'apprenti-danseur compte ses pas, mais non le danseur, qui les fait ou bien qui est porté et poussé par eux.

« Etre », c'est être poussé dehors, c'est-à-dire ex-ister. Chaque existant est un existant - ce qui ne veut pas dire qu'il soit un en soi et pour soi, unique et uni - mais la pulsation qui projette tous et chacun relève d'une unité bien différente. En ce sens le monde est bien « un » mais cette unité nous est dérobée par le nombre - non pas toutefois par la grandeur du nombre, bien que cette grandeur elle-même semble se complaire à s'additionner pour s'additionner - mais par le fait que sous la numération et la multitude nous peinons à comprendre quelle unité pourrait se révéler.

Sans doute ne se révèle-t-elle pas, au sens qu'on donne à ce terme. Elle ne vient pas au jour, elle ne se laisse ni connaître, ni compter. Mais elle s'avère toujours et partout. Toujours et partout en effet, dans la profusion exorbitante de la nature comme dans la prolifération voire le pullulement de nos machines, de nos signaux, de nos fins sans finalité, toujours et partout s'avère d'autant plus quelque chose comme « un » envoi - non pas un projet mais un jet, une éjection du monde (par nous, en nous) vers un dehors toujours plus vaste et toujours plus retiré à l'assurance d'une unité de dessein, de visée, d'histoire.

Il se peut en effet que l'Un antérieur à toute unité soit retiré non pas seulement à notre prise - ce qu'il fut toujours comme toujours nous l'avons su - mais à luimême. Cela se dit « Dieu est mort».

Mais une autre face de ce retrait pourrait être que l'Un antérieur, l'un éminent ou " maxime unum » comme dit Thomas d'Aquin ${ }^{8}$ se retire de toute unité et de toute numération. Se retirant, et laissant en effet la dépouille vide du " un », il indique une unité plus haute, incalculable, innumérable - innombrable donc aussi et prenant en charge l'innombrable des grands nombres - mais « une » en ce qu'el-

8 Summa Theologica, Ia, qu.11. 
le excède toute multiplicité, toute diversité mais par là-même toute unité comptable dans une multiplicité.

Un d'au-delà ou d'avant l'Un. Un ponctuel, sans dimension, n'ayant pas lieu, ni lieu ni temps mais ouvrant toute possibilité de temps et de lieu. Cet excès n'a rien de magico-mystique et il ne s'agit pas d'une entité métaphysique brumeuse : l'excès est donné dans la nature de " un ». Si " l'être et l'un se convertissent l'un en l'autre » comme le voulait la doctrine classique des transcendantaux, et si « être » c'est être poussé dehors (dehors, devant, plus loin, ailleurs, comme on voudra), alors « un » donne l'énergie de cette poussée, et il la donne en tant que pulsation rythmique.

Le « maxime unum », l'un le plus grand qui soit, est plus grand en ce qu'il est " maxime indivisum » comme dit aussi Thomas. Etre suprêmement indivis c'est n'être susceptible de la division en aucune manière, ni en parties, ni en substance et accidents, ni en acte et puissance, ni pour finir en " quelque chose » et la nature, la position ou l'existence de cette chose. L'un excédent, s'excédant lui-même, se retire de toute assignation de ce genre et ne peut être considéré que comme cette unité et unicité de la pulsation, pulsion, impulsion. Ce n'est pas un « un », ce n'est que la simplicité absolue d'une poussée, d'un bond, d'un élan.

Son unicité est absolue, et c'est pourquoi il est aussi bien « un et tout » selon cette unité du tout dont on attribue la pensée à Héraclite qui est aussi celui dont nous avons le mot «l'un s'ajointe à soi en différant de soi ». L'un excédant « est » tout précisément en différant de soi, en se différant dans la poussée qui ouvre, écarte et disperse toutes choses. Mais pour autant il ne se différencie pas de lui-même ni ne se résout en différences. Il se diffère - ce qu'il faut entendre selon la logique de la différance : ce différer ne lui survient pas, mais il est bien plutôt lui-même ce différer. Il « est », si on peut dire, il n'est que mais il est intégralement le mouvement, la poussée, la pulsion qui se porte et qui porte en avant, qui se transporte et se communique à toute existence, à toutes les existences. Il ne faut pas négliger le fait que dans le fameux « un différant de soi » d'Héraclite le verbe diapherô doit être aussi ou d'abord compris selon sa valeur de transport, de mouvement et d'ébranlement qui emporte.

L'un excédant est un emportement. Celui du fait même qu'il y ait un monde, ou des mondes, autant qu'on voudra, qu'il y ait quelque chose. C'est l'un secret de ce « quelque chose » indéterminé, anonyme et discret dont la discrétion éclate en même temps en multiplicités indéfinies d'unités et d'unicités. On peut dire, suivant ainsi la logique de la différance, que cet emportement n'emporte rien qui lui aurait préexisté, pas même lui-même9.

${ }^{9}$ C'est aujourd'hui la pensée de la science qui se détourne de l'unification : "Ne faudrait-il pas mieux renoncer à la prolifération des mondes et à l'évocation de versions correctes constituant chacune un monde pour revenir à l'image plus orthodoxe de différentes descriptions d'un seul et même monde 
Cet un est un autre « plus d'un ». Il est - pour autant qu'il « soit»- le « plus d'un » qui tourne en « plus qu'un »: plus que « un » mais pas plus nombreux, au contraire plus un que un : non pas compte mais transport, non pas unité mais envoi, non pas donné mais donnant. Unique, mais non isolé, repris au contraire et relancé en toute scansion d'existence, dont chacune est unique. Un de simplicité absolue et non d'union : il ne réunit rien en lui puisqu'il ouvre le pas - le passage, la bande passante - du divers, le pas lui-même divisé de la division de toutes choses.

Comme l'écrit Blanchot : « Le «Un » est ce qui autorise le moins l'union, fûtce avec l'infiniment lointain, à plus forte raison la remontée et la confusion mystiques. » 10 Pas de confusion, mais la profusion. Le un qui n'est pas ajouté - ou « pas seulement » ajouté, et qui transforme le sens même de l' « ajout »- et qui n'est pas non plus réuni en soi, le un ni additif ni inclusif n'est pas pour autant retiré en luimême à la manière d'un point (tel que l'est le "Je » kantien, par exemple) : au contraire il est ce transport de lui-même et hors de lui-même (mais il n'a ni dehors, ni dedans), transport du point, en un sens, mais alors transport qui fait ligne, non pas continue mais rythmique, cadence de la pluralité toujours renouvelée de tous les singuliers. Chaque singulier est lui-même « un » en ce sens : c'est-à-dire en ce qu'il répète le transport initial - transport de l'initial - étant lui-même à son tour ouverture d'autres profusions (une vie, par exemple, tous les moments, toutes les allures, tous les heurts d'une vie, et sa mort).

S'il y a une unité du monde, ou plus exactement si le «monde » peut être pensé comme l'unité non numérisable de l'être, de cet être compris comme singularité rythmique et selon cette « essence ternaire » de l'infini dont parlait Derrida, il faut qu'elle soit de cet ordre. C'est-à-dire de l'ordre de la profusion des espèces, des aspects et des manières de l'existant, de la poussée prolifique des vivants et de cette autre prolifération qui est celle de nos machines, appareils, engins, instruments, moteurs, organes greffés, transorganiques, virtuels, fiduciaires, fictifs.

Mais il faut pour cela que la prolifération et la profusion soient abondance et non accumulation par addition. Ni addition d'un moment du temps à un autre, ni addition d'une portion d'espace à une autre, ni d'un individu à un autre. Nous savons que la forme générale la plus abstraite de cette addition est la monnaie ou l'argent : l'équivalence et la permutabilité d'unités cumulables (de " numéraires » a-t-on dit naguère). Nous savons aussi que c'est le développement exponentiel de

neutre et sous-jacent? Mais le monde ainsi reconquis, écrit Goodman, « n'a plus ni genre ni ordre, ni mouvement, ni repos, ni structure -c'est un monde qui ne mérite pas qu'on lutte pour ou contre lui ». C'est l'hypothèse de trop. Cette "passion de l'un », comme la nomme Goodman, du sous-jacent, du fondamental, de l'Etre ou de l'arche atrophie les possibles. Elle ne subsume pas nécessairement sous un maître-concept, elle ferme aussi des voies d'accès et des canaux d'irrigation. Elle élague les ramures et endommage les radicelles. " Aurélien Barrau, La cosmologie comme " manière de faire un monde »-Physique, relativisme et irréalisme - Université de Grenoble, CNRS IN2P3, 2009.

10 L'Écriture du désastre, Paris, Gallimard, 1980, p. 211. 
ses techniques (crédit, actions, banques, etc.) qui a rendu possible le déploiement des autres techniques et avec elles de notre entrée dans l'ère des grands nombres cosmiques, démographiques, électroniques - dont nous ne savons pas s'ils valent comme pullulement, pléthore, engorgement, ou bien comme abondance, générosité, richesse - mais en quel sens de la richesse ?

Richesse, cela peut faire un autre mot pour " plus d'un ». Et cela nous expose très clairement la croisée des chemins : entre l'accumulation des unités décomptables et une générosité qui ne peut venir que d'ailleurs et d'avant, de l'unité d'un rythme infini. Voilà peut-être pourquoi notre civilisation se préoccupe tant de l'un depuis le début : parce qu'elle sait qu'elle s'est engagée entre les deux, entre l'un qui s'ajoute à lui-même et l'un qui s'emporte hors de soi.

Entre l'un et l'autre : nous savons aujourd'hui que nous avons à reprendre tous les termes de cette alternative, ou de cet intervalle, de cette contradiction ou de cette complication. Entre l'un et l'autre un. Ce qui implique aussitôt : entre l'un et l'autre. L'autre est-il l'autre un ou bien l'autre de l'un ? Et duquel ? A suivre. 